\title{
Pacific
}

Journal of

Mathematics

\section{REALIZING THE LOCAL WEIL REPRESENTATION OVER A NUMBER FIELD}

Gerald ClifF AND DAVID MCNEILly 


\title{
REALIZING THE LOCAL WEIL REPRESENTATION OVER A NUMBER FIELD
}

\author{
GeRAld ClifF AND DAVID MCNEILly
}

Let $F$ be a non-Archimedean local field whose residue field has order $q$ and characteristic $p \neq 2$. We show that the Weil representations of the symplectic group $\operatorname{Sp}(2 n, F)$ can be realized over the field

$$
E_{0}= \begin{cases}\mathbb{Q}(\sqrt{p}, \sqrt{-p}), & \text { if } q \text { is not a square; } \\ \mathbb{Q}(\sqrt{-p}), & \text { if } q \text { is a square and } p \equiv 1 \bmod 4 ; \\ \mathbb{Q}(\sqrt{-1}), & \text { if } q \text { is a square and } p \equiv 3 \bmod 4 .\end{cases}
$$

Furthermore, the field $E_{0}$ is shown to be optimal if $q \equiv 1 \bmod 4$.

\section{Introduction}

Let $F$ be a non-Archimedean local field whose residue field has order $q$ and characteristic $p \neq 2$. Our main result is that the Weil representations of the symplectic group $\operatorname{Sp}(2 n, F)$, can be realized over the number field

$$
E_{0}= \begin{cases}\mathbb{Q}(\sqrt{p}, \sqrt{-p}), & \text { if } q \text { is not a square; } \\ \mathbb{Q}(\sqrt{-p}), & \text { if } q \text { is a square and } p \equiv 1 \bmod 4 ; \\ \mathbb{Q}(\sqrt{-1}), & \text { if } q \text { is a square and } p \equiv 3 \bmod 4 .\end{cases}
$$

This answers a question raised by D. Prasad [1998]. A consequence of this, also pointed out by Prasad, is that the local theta correspondence can be defined for representations which are realized over $E_{0}$.

Let $\lambda$ be a nontrivial, continuous, complex, unitary character of the additive group of the field $F$. We shall use $\mathbb{Q}(\lambda)$ to denote the field obtained by adjoining all of the character values of $\lambda$ to $\mathbb{Q}$, and set $E=\mathbb{Q}(\lambda)(\sqrt{-1})$. We observe that $E$ is an algebraic extension of $\mathbb{Q}$. Indeed, if $F$ has characteristic $0, E$ is the field obtained from $\mathbb{Q}$ by adjoining $\sqrt{-1}$ and all $p$-power roots of unity. On the other hand, if char $F=p$ then $E$ is the number field obtained by adjoining a primitive $4 p$-th root of unity to $\mathbb{Q}$.

MSC2000: 11F70, 22E50.

Keywords: Weil representation, local fields. 
Let $F^{2 n}=X \oplus Y$ be a decomposition of $F^{2 n}$ as a direct sum of totally isotropic $F$ subspaces, with respect to the alternating form on $F^{2 n}$ used to define the symplectic group $\operatorname{Sp}(2 n, F)$. Ranga Rao [1993] provided an explicit realization of the Weil representation $W_{\lambda}$ of $\operatorname{Sp}(2 n, F)$ associated with $\lambda$ as integral operators acting on the Bruhat-Schwartz space $\mathscr{Y}(X)$ of complex valued, locally constant functions on $X$ of compact support. For a subfield $L$ of $\mathbb{C}$, define $\mathscr{S}(X, L)$ to be the space of locally constant functions on $X$ of compact support having values in $L$. Observing that the Haar measure $\mu_{\lambda, g}$ used to define the operators $W_{\lambda}(g)$ is $\mathbb{Q}(\sqrt{q})$-rational, we are able to show that the space $\mathscr{S}(X, E)$ is invariant under the Weil representation $W_{\lambda}$, hence provides a realization of the Weil representation over the algebraic extension $E$. In particular, this provides an affirmative answer to Prasad's question in the case $\operatorname{char} F=p$.

The latter half of the paper is devoted to the construction a 1-cocycle $\delta$ on $\operatorname{Gal}\left(E / E_{0}\right)$ with values in $\operatorname{GL}(\mathscr{S}(X, E))$ such that

$$
{ }^{\sigma} W_{\lambda}(g)=\delta(\sigma)^{-1} W_{\lambda}(g) \delta(\sigma), \quad g \in \operatorname{Sp}(V) .
$$

Using Galois descent, we show that there exists $\alpha \in \mathrm{GL}(\mathscr{Y}(X, E))$ such that $\delta(\sigma)=$ $\alpha^{-1 \sigma} \alpha$ for $\sigma \in \operatorname{Gal}\left(E / E_{0}\right)$.

Main theorem. The operators $\alpha W_{\lambda}(g) \alpha^{-1}$ leave $\mathscr{S}\left(X, E_{0}\right)$ invariant, and provide a form of the Weil representation realized over $E_{0}$.

We should remark that we fail to provide an explicit description of the operator $\alpha$. As such, the problem of finding an explicit realization of the Weil representation over $E_{0}$ remains open.

To indicate how we find the 1-cocycle satisfying (I), for the rest of the introduction we assume that $F$ has characteristic 0 . The Galois group of $\mathbb{Q}(\lambda) / \mathbb{Q}$ is isomorphic to the units $\mathbb{Z}_{p}^{*}$ of the $p$-adic integers. For an element $s$ of $\mathbb{Z}_{p}^{*}$, we let $\sigma_{s}$ denote the corresponding element of $\operatorname{Gal}(\mathbb{Q}(\lambda) / \mathbb{Q})$. For an element $t \in F^{*}$, we define the character $\lambda[t]$ of $F$ by $\lambda[t](r)=\lambda(t r), r \in F$.

For $t \in F^{*}$, let $g_{t} \in \operatorname{Sp}(2 n, F)$ and $f_{t} \in \mathrm{GL}(2 n, F)$ be defined by

$$
\begin{aligned}
& (x+y) g_{t}=t^{-1} x+t y, \\
& (x+y) f_{t}=x+t y,
\end{aligned}
$$

where $x \in X, y \in Y$. In general, $f_{t}$ is not in $\operatorname{Sp}(2 n, F)$, but conjugation by $f_{t}$ leaves $\operatorname{Sp}(2 n, F)$ invariant. We have

$$
W_{\lambda}\left(g^{f_{t}}\right)=W_{\lambda[t]}(g), \quad g \in \operatorname{Sp}(V) .
$$

Furthermore, observing $f_{t^{2}}$ is the composite $t I \circ g_{t}$, we show

$$
W_{\lambda}\left(g^{f_{t^{2}}}\right)=W_{\lambda}\left(g_{t}\right)^{-1} W_{\lambda}(g) W_{\lambda}\left(g_{t}\right) .
$$


On the other hand, restriction to $\mathbb{Q}(\lambda)$ identifies $\operatorname{Gal}\left(E / E_{0}\right)$ with $\left(F^{*}\right)^{2} \cap \mathbb{Z}_{p}^{*}$. If $\sigma \in \operatorname{Gal}\left(E / E_{0}\right)$, we can write

$$
\left.\sigma\right|_{\mathbb{Q}(\lambda)}=\sigma_{t^{2}}
$$

for some $t \in F^{*}$. We note

$$
{ }^{\sigma} W_{\lambda}(g)=W_{\lambda\left[t^{2}\right]}(g) .
$$

In light of (II) and (III), we deduce the fundamental identity

$$
{ }^{\sigma} W_{\lambda}(g)=W_{\lambda}\left(g_{t}\right)^{-1} W_{\lambda}(g) W_{\lambda}\left(g_{t}\right) .
$$

The last equation is used to show that $\delta(\sigma)=W_{\lambda}\left(g_{t}\right)$ satisfies (I) and almost satisfies the one-cocycle condition. An actual one-cocycle is obtained by slightly modifying the operators $W_{\lambda}\left(g_{t}\right)$.

The paper concludes with an investigation of the optimality of the field $E_{0}$. Our main tool is the $K$-types associated with the compact subgroup $\operatorname{Sp}(\mathscr{L})$ of elements preserving a lattice $\mathscr{L}$ on which the symplectic form $\langle$,$\rangle is nondegenerate. If$ $q \equiv 1 \bmod 4$ then it is impossible to realize the $K$-types in a proper subfield of $E_{0}$, which allows us to deduce that $E_{0}$ is optimal for realizing $W_{\lambda}$. If $q \equiv 3 \bmod 4$, the $K$-types can be realized over the proper subfield $\mathbb{Q}(\sqrt{-p})$ of $E_{0}$. In this case, the possibility of realizing the Weil representation over the smaller field is left open.

\section{Preliminary remarks on local fields, characters and measures}

We fix some notation and recall some elementary facts about the characters of the additive group of a local field. Further details can be found in the first two chapters of [Weil 1974].

Let $F$ be a non-Archimedean local field, $O$ its ring of integers, and $\mathfrak{m}$ the maximal ideal of 0 . The order of the residue class field $\kappa=0 / \mathfrak{m}$ shall be denoted $q$; we note that $q$ is power of $p=$ char $\kappa$. We assume throughout that $p$ is different from 2 ; in particular, 2 is a unit of 0 .

Given a fractional $\mathbb{O}$-ideal $\mathfrak{a}$, there exists an unique integer $v(\mathfrak{a})$, the valuation of $\mathfrak{a}$, such that

$$
\mathfrak{a}=\mathfrak{m}^{v(\mathfrak{a})} .
$$

If $s \in F$ is nonzero, the valuation of the ideal $s \mathbb{O}$ is referred to as the valuation of $s$, denoted $v(s)$. The absolute value on $F$ is related to the valuation $v$ on $F$ by

$$
|s|=q^{-v(s)}, \quad s \in F, s \neq 0 .
$$

Let $\lambda$ be a nontrivial, continuous, unitary, complex linear character of $F^{+}$. The continuity of $\lambda$ ensures that its kernel contains a fractional 0 -ideal. The fact that $\lambda$ is nontrivial allows one to deduce that the set of all such fractional $\mathrm{O}$-ideals has a 
unique maximal element $\mathfrak{i}=\mathfrak{i}_{\lambda}$, the conductor of $\lambda$. The level of $\lambda$ is defined to be the valuation of $\mathfrak{i}_{\lambda}$.

Given $n \geq 1$, let

$$
v_{p^{n}}=\left\{z \in \mathbb{C}: z^{p^{n}}=1\right\}, \quad v_{p^{\infty}}=\bigcup_{n=1}^{\infty} v_{p^{n}} .
$$

(The more customary symbol $\mu$ will be used to denote a measure.)

Lemma 1. We have

$$
\operatorname{im} \lambda= \begin{cases}v_{p} & \text { if } \operatorname{char} F=p, \\ v_{p^{\infty}} & \text { if } \operatorname{char} F=0 .\end{cases}
$$

Proof. Take $x \in F$. If $\operatorname{char} F=p$ then

$$
1=\lambda(0)=\lambda(p x)=\lambda(x)^{p} .
$$

This shows $\operatorname{im} \lambda \subseteq v_{p}$. Equality follows from the fact $\operatorname{im} \lambda$ is a nontrivial subgroup of the simple abelian group $v_{p}$.

If $\operatorname{char} F=0$ then, since $p \in \mathfrak{m}$, there exists an $n \geq 0$ such that $p^{n} x \in \mathfrak{i}_{\lambda}$. For such $n$,

$$
1=\lambda\left(p^{n} x\right)=\lambda(x)^{p^{n}} .
$$

Then $\operatorname{im} \lambda \subseteq v_{p^{\infty}}$. If the inclusion were proper then there would exist $m \geq 0$ such that $\operatorname{im} \lambda=v_{p^{m}}$. In this case, if $x \in F$ then

$$
\lambda(x)=\lambda\left(p^{m} \cdot \frac{x}{p^{m}}\right)=\lambda\left(\frac{x}{p^{m}}\right)^{p^{m}}=1
$$

since $\lambda\left(x / p^{m}\right)$ is a $p^{m}$-th root of unity. As this would contradict the nontriviality of $\lambda, \operatorname{im} \lambda=v_{p^{\infty}}$.

Define $\mathbb{Q}(\lambda)$ to be the field obtained by adjoining to $\mathbb{Q}$ all the character values $\lambda(x), x \in F$. Define

$$
\mathscr{P} \simeq \begin{cases}\mathbb{Z} / p \mathbb{Z} & \text { if } \operatorname{char} F=p, \\ \mathbb{Z}_{p} & \text { if } \operatorname{char} F=0 .\end{cases}
$$

Note that $\mathscr{P}$ is the topological closure of the prime ring of $F$.

Lemma 2. There is a canonical topological isomorphism

$$
\operatorname{Gal}(\mathbb{Q}(\lambda) / \mathbb{Q}) \simeq \mathscr{P}^{*}
$$


Proof. The preceding lemma ensures that $\operatorname{im} \lambda$ is invariant under the action of Galois, hence restriction yields a homomorphism

$$
\operatorname{Gal}(\mathbb{Q}(\lambda) / \mathbb{Q}) \rightarrow \operatorname{Aut}(\operatorname{im} \lambda) \simeq \begin{cases}(\mathbb{Z} / p \mathbb{Z})^{*} & \text { if } \operatorname{char} F=p, \\ \mathbb{Z}_{p}^{*} & \text { if } \operatorname{char} F=0 .\end{cases}
$$

It is readily checked that this map is an isomorphism of topological groups. The proof is completed by appealing to the description of $\mathscr{P}$ given above.

The pairing

$$
(s, t) \rightarrow \lambda(s t), \quad s, t \in F,
$$

is nondegenerate and leads to an identification of $F^{+}$with its Pontryagin dual [Weil 1974, II.5]. The image of $s \in F$ in the dual shall be denoted $\lambda[s]$ :

$$
\lambda[s](t)=\lambda(s t), \quad t \in F .
$$

Let $\mu=d t$ be a Haar measure on $F^{+}$. If $\phi$ is a locally constant, complex valued function on $F$ of compact support, the Fourier transform $\mathscr{F}_{\lambda} \phi$ is the complex valued function on $F$ defined by

$$
\mathscr{F}_{\lambda} \phi(s)=\int_{F} \lambda[s](t) \phi(t) d t, \quad s \in F .
$$

It can be shown that $\mathscr{F}_{\lambda} \phi$ is locally constant and has compact support. Furthermore, the general theory of Fourier transforms asserts the existence of a positive constant $c$, depending only on the Haar measure $d t$, such that

$$
\left(\mathscr{F}_{\lambda} \mathscr{F}_{\lambda} \phi\right)(t)=c \phi(-t), \quad t \in F .
$$

There is a unique Haar measure on $F^{+}$for which $c=1$; it shall be denoted $d_{\lambda} t$ and will be referred to as the self-dual Haar measure associated with $\lambda$ [Weil 1974, VII.2].

Lemma 3. If $\lambda$ has level $l$ then the associated self-dual Haar measure is characterized by the condition

$$
\int_{0} d_{\lambda} t=q^{l / 2}
$$

Proof. This follows from [Weil 1974, Corollary 3, VII.2].

Corollary. If $s \in F^{*}$ then

$$
d_{\lambda[s]} t=|s|^{1 / 2} d_{\lambda} t .
$$

Proof. Since $\mathfrak{i}_{\lambda}=s \mathfrak{i}_{\lambda[s]}$, the levels $l_{1}$ of $\lambda$ and $l_{2}$ of $\lambda[s]$ satisfy the relation $l_{1}=$ $v(s)+l_{2}$. Therefore, Lemma 3 yields

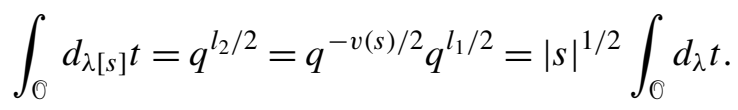




\section{The Schrödinger and Weil representations}

Let $\langle$,$\rangle be a nondegenerate, alternating, F$-bilinear form on a finite dimensional $F$-vector space $V$. The Heisenberg group $H$ is the group on $V \times F$ having multiplication

$$
(v, t)\left(v^{\prime}, t^{\prime}\right)=\left(v+v^{\prime}, t+t^{\prime}+\left\langle v, v^{\prime}\right\rangle / 2\right), \quad t, t^{\prime} \in F, v, v^{\prime} \in V .
$$

Let $\lambda$ be a nontrivial, continuous, unitary, complex linear character of $F^{+}$. Since $Z(H)=0 \times F \simeq F^{+}$, it may be viewed as a character of the center of the Heisenberg group $H$.

Theorem (Stone, von Neumann). There exists a smooth, irreducible representation of $H$ having central character $\lambda$. Such a representation is necessarily admissible, and is unique up to isomorphism.

A proof of the Stone-von Neumann Theorem can be found in [Mœglin et al. 1987, 2.I]. The representation provided by the Stone-von Neumann Theorem is referred to as the Schrödinger representation of type $\lambda$.

The symplectic group

$$
\operatorname{Sp}(V)=\{g \in \mathrm{GL}(V):\langle v g, w g\rangle=\langle v, w\rangle, v, w \in V\}
$$

acts on the Heisenberg group $H$ as a group of automorphisms as follows: if $g \in$ $\operatorname{Sp}(V)$ and $(t, v) \in H$ then

$$
(t, v) g=(t, v g) .
$$

Given a Schrödinger representation $S_{\lambda}$ of type $\lambda$ and $g \in \operatorname{Sp}(V)$, consider the representation $S_{\lambda}^{g}$ of $H$ defined by

$$
S_{\lambda}^{g}(h)=S_{\lambda}(h g), \quad h \in H .
$$

It is readily verified that $S_{\lambda}^{g}$ is a smooth, irreducible representation of $H$. Furthermore, observing that $g$ acts trivially on $Z(H), S_{\lambda}^{g}$ has central character $\lambda$. The Stone-von Neumann Theorem allows us to conclude that the representation $S_{\lambda}$ and $S_{\lambda}^{g}$ are equivalent, hence the ambient space affording $S_{\lambda}$ admits an operator $W_{\lambda}(g)$ for which

$$
S_{\lambda}^{g}(h)=W_{\lambda}(g)^{-1} S_{\lambda}(h) W_{\lambda}(g), \quad h \in H .
$$

In light of Schur's Lemma, the operator $W_{\lambda}(g)$ is uniquely defined up to multiplication by a nonzero constant. As a result, the map

$$
g \mapsto W_{\lambda}(g), \quad g \in \operatorname{Sp}(V),
$$

is a projective representation of $\operatorname{Sp}(V)$, called a Weil representation of type $\lambda$. 
In this paper we consider the Schrödinger models of $S_{\lambda}$ and $W_{\lambda}$ [Kudla 1996, Lemma 2.2, Proposition 2.3; Mœglin et al. 1987, 2.I.4(a) and 2.II.6; Ranga Rao 1993, §3]. Let

$$
V=X+Y
$$

where $X$ and $Y$ are maximal, totally isotropic subspaces. The Schrödinger model is realized in the Bruhat-Schwartz space $\mathscr{S}(X)$ of locally constant functions $f: X \rightarrow \mathbb{C}$ of compact support: if $x \in X, y \in Y$ and $t \in F$ then $S_{\lambda}((x+y, t))$ is the operator defined by

$$
\left[S_{\lambda}((x+y, t)) \phi\right]\left(x^{\prime}\right)=\lambda\left(t+\frac{\langle x, y\rangle}{2}+\left\langle x^{\prime}, y\right\rangle\right) \phi\left(x+x^{\prime}\right), \quad \phi \in \mathscr{Y}(X), x^{\prime} \in X .
$$

The description of the Weil representation requires some additional notation. Viewing $x+y \in V$ as a row vector $(x, y)$, each $g \in \operatorname{Sp}(V)$ can be expressed in the matrix form

$$
g=\left(\begin{array}{ll}
a & b \\
c & d
\end{array}\right)
$$

where $a: X \rightarrow X, b: X \rightarrow Y, c: Y \rightarrow X$, and $d: Y \rightarrow Y$. With this notation, set

$$
Y_{g}=Y / \operatorname{ker} c .
$$

If $\mu_{g}$ is a Haar measure on $Y_{g}$ then the action of $W_{\lambda}(g)$ on $\mathscr{S}(X)$ is given by

$$
\left[W_{\lambda}(g) \phi\right](x)=\int_{Y_{g}} \lambda\left(\frac{\langle x a, x b\rangle-2\langle x b, y c\rangle+\langle y c, y d\rangle}{2}\right) \phi(x a+y c) d \mu_{g} y,
$$

for $\phi \in \mathscr{S}(X)$ and $x \in X$. Note that the integral appearing in (3) is well-defined, for the integrand is constant on the cosets of $\operatorname{ker} c$, hence can be viewed as a function on $Y_{g}$. The fact $\phi \in \mathscr{T}(X)$ can be used to show that the integrand belongs to $\mathscr{Y}\left(Y_{g}\right)$, hence the integral converges, and that the resulting function $W_{\lambda}(g) \phi$ belongs to $\mathscr{Y}(X)$.

We now recall a particular choice of Haar measures $\mu_{\lambda, g}$ on $Y_{g}, g \in \mathrm{Sp}(V)$ [Ranga Rao 1993, §3.3]. Fix a basis $x_{1}, \ldots, x_{n}$ of $X$ and let $y_{1}, \ldots, y_{n}$ be the dual basis of $Y$ defined by the conditions

$$
\left\langle x_{i}, y_{j}\right\rangle=\delta_{i j}, \quad 1 \leq i, j \leq n .
$$

Let $\tau_{i}, 0 \leq i \leq n$, be the element of $\operatorname{Sp}(V)$ defined by

$$
\begin{aligned}
& x_{j} \tau_{i}=\left\{\begin{aligned}
-y_{j} & \text { if } j \leq i, \\
x_{j} & \text { if } i<j,
\end{aligned}\right. \\
& y_{j} \tau_{i}=\left\{\begin{aligned}
x_{j} & \text { if } j \leq i, \\
y_{j} & \text { if } i<j .
\end{aligned}\right.
\end{aligned}
$$


We note that $Y_{\tau_{i}}$ can be identified with the subspace of $Y$ spanned by the elements $y_{1}, \ldots, y_{i}$. We define

$$
d \mu_{\lambda, \tau_{i}} y=\prod_{k=1}^{i} d_{\lambda} y_{k}
$$

where $d_{\lambda} y_{k}$ is the self-dual Haar measure associated with $\lambda$.

Let

$$
P=\{g \in \mathrm{Sp}(V): Y g=g\},
$$

the parabolic subgroup that leaves $Y$ invariant. If $\operatorname{dim} Y_{g}=i$ then [Ranga Rao 1993, Theorem 2.14] ensures the existence of elements $p_{1}$ and $p_{2}$ of $P$ such that

$$
g=p_{1} \tau_{i} p_{2}
$$

Observing that the operator $p_{1}$ induces an isomorphism $\bar{p}_{1}: Y_{g} \rightarrow Y_{\tau_{i}}$, we set

$$
\mu_{\lambda, g}=\left|\operatorname{det}\left(\left.p_{1} p_{2}\right|_{Y}\right)\right|^{-1 / 2} \bar{p}_{1} \cdot \mu_{\lambda, \tau_{i}} .
$$

Here, $\bar{p}_{1} \cdot \mu_{\lambda, \tau_{i}}$ denotes the pullback of the Haar measure $\mu_{\lambda, \tau_{i}}$ to $Y_{g}$ via $\bar{p}_{1}$ : if $E$ is a measurable subset of $Y_{g}$ then

$$
\bar{p}_{1} \cdot \mu_{\lambda, \tau_{i}}(O)=\mu_{\lambda, \tau_{i}}\left(O \bar{p}_{1}\right) .
$$

Theorem 4. The measures $\mu_{\lambda, g}, g \in S p(V)$, are well-defined. The projective representation $W_{\lambda}$ of $\operatorname{Sp}(V)$ defined by (3) with the Haar measures $\mu_{g}=\mu_{\lambda, g}$ has the following properties.

(i) If $g \in \operatorname{Sp}(V)$ and $p_{1}, p_{2} \in P$ then $W_{\lambda}\left(p_{1} g p_{2}\right)=W_{\lambda}\left(p_{1}\right) W_{\lambda}(g) W_{\lambda}\left(p_{2}\right)$; in particular $W_{\lambda}$ restricts to an ordinary representation of $P$.

(ii) If $\phi \in \mathscr{Y}(X)$ and $p=\left(\begin{array}{ll}a & b \\ 0 & d\end{array}\right) \in P$ then

$$
\left[W_{\lambda}(p) \phi\right](x)=|\operatorname{det} a|^{1 / 2} \lambda\left(\frac{\langle x a, x b\rangle}{2}\right) \phi(x a), \quad x \in X .
$$

Proof. This follows from [Ranga Rao 1993, Theorem 3.5].

Lemma 5. If $s \in F^{*}$ and $g \in \mathrm{Sp}(V)$ then $\mu_{\lambda[s], g}=|s|_{Y_{g}}^{1 / 2} \mu_{\lambda, g}$.

Proof. In light of the Corollary to Lemma 3, (4) yields

$$
d \mu_{\lambda[s], \tau_{i}} y=\prod_{k=1}^{i} d_{\lambda[s]} y_{k}=\prod_{k=1}^{i}\left[|s|^{1 / 2} d_{\lambda} y_{k}\right]=|s|^{i / 2} \prod_{k=1}^{i} d_{\lambda} y_{k}=|s|^{i / 2} d \mu_{\lambda, \tau_{i}} y .
$$

Therefore, we obtain from (5) and the fact that $Y_{g}$ has dimension $i$ over $F$ that

$$
\begin{aligned}
\mu_{\lambda[s], g} & =\left|\operatorname{det}\left(\left.p_{1} p_{2}\right|_{Y}\right)\right|^{-1 / 2} \bar{p}_{1} \cdot \mu_{\lambda[s], \tau_{i}}=|s|^{i / 2}\left|\operatorname{det}\left(\left.p_{1} p_{2}\right|_{Y}\right)\right|^{-1 / 2} \bar{p}_{1} \cdot \mu_{\lambda, \tau_{i}} \\
& =|s|^{i / 2} \mu_{\lambda, g}=|s|_{Y_{g}}^{1 / 2} \mu_{\lambda, g}
\end{aligned}
$$


Let $\mu$ be a Haar measure on a totally disconnected topological group $A$. If $O_{1}$ and $\mathrm{O}_{2}$ are nonempty compact open sets in $A$ then the ratio

$$
\left(O_{1}: O_{2}\right)=\frac{\mu\left(O_{1}\right)}{\mu\left(O_{2}\right)}
$$

is a rational number [Cartier 1979, I.1.1]. Hence, if $\mu(O)$ lies in a subfield $L$ of $\mathbb{C}$ for some nonempty compact open set $O$ then the same is true for all nonempty compact open sets. The measure $\mu$ is said to L-rational if this is the case.

Lemma 6. The measures $\mu_{\lambda, g}, g \in \operatorname{Sp}(V)$, are $\mathbb{Q}(\sqrt{q})$-rational.

Proof. If $t \in F^{*}$ then $|t|$ is a power of $q$. Therefore, (5) shows that it is sufficient to verify that the measures $\mu_{\lambda, \tau_{i}}$ are $\mathbb{Q}(\sqrt{q})$-rational. Formulas (1) and (4) ensure that this is indeed the case: if $\mathscr{y}_{i}=\sum_{k=1}^{i} O y_{k}$ then

$$
\int_{\mathscr{\vartheta}_{i}} d \mu_{\lambda, \tau_{i}} y=q^{i l / 2} \text {. }
$$

If $L$ is a subfield of $\mathbb{C}$, let $\mathscr{S}(A, L)$ denote the space of locally constant, $L$-valued functions on $A$ of compact support.

Lemma 7. Let $A$ be a totally disconnected topological group, $L \subseteq K$ an extension of fields, and $\mu$ a L-rational Haar measure on A. If $\phi \in \mathscr{S}(A, K)$ then $\int_{A} \phi d \mu$ belongs to $K$.

Proof. Since $\phi \in \mathscr{Y}(A, K)$, there exists compact open subsets $A_{1}, \ldots, A_{k}$ of $A$ and scalars $c_{1}, \ldots, c_{k}$ in $K$ such that

$$
\phi=\sum_{i=1}^{k} c_{i} \chi_{A_{i}} .
$$

Here, $\chi_{A_{i}}$ denotes the characteristic function of $A_{i}$. Since $\mu\left(A_{i}\right) \in L \subseteq K$, it follows that

$$
\int_{A} \phi d \mu=\sum_{i=1}^{k} c_{i} \mu\left(A_{i}\right)
$$

lies in $K$.

Let $\mathbb{Q}(\lambda)$ be the character field of $\lambda$ and set

$$
E=\mathbb{Q}(\lambda)(\sqrt{-1}) .
$$

Observe that Lemma 1 ensures that $\mathbb{Q}(\sqrt{q})$ is a subfield of $E$.

Proposition 8. The operators $W_{\lambda}(g), g \in \operatorname{Sp}(V)$, leave the subspace $\mathscr{S}(X, E)$ invariant. 
Proof. If $\phi \in \mathscr{Y}(X, E)$ then the integrand in (3) lies in $\mathscr{Y}\left(Y_{g}, E\right)$, since $\mathbb{Q}(\lambda) \subseteq E$. In light of Lemma 6, Lemma 7 applied in the case $A=Y_{g}, K=E, L=\mathbb{Q}(\sqrt{q})$, and $\mu=\mu_{\lambda, g}$ allows us to deduce that the integral (3) lies in $E$. It follows immediately that $W_{\lambda}(g) \phi \in \mathscr{Y}(X, E)$.

In particular, if $F$ has odd characteristic $p$, the preceding result allows one to conclude that the Weil representation $W_{\lambda}$ can be realized over the number field $\mathbb{Q}\left(v_{4 p}\right)$.

\section{Galois action}

By Lemma $1, E$ is a Galois extension of $\mathbb{Q}$. Its Galois group acts on $\mathscr{Y}(X, E)$ : if $\sigma \in \operatorname{Gal}(E / \mathbb{Q})$ and $\phi \in \mathscr{Y}(X, E)$ then

$$
(\sigma(\phi))(x)=\sigma(\phi(x)), \quad x \in X .
$$

There is an associated Galois action on End $\mathscr{Y}(X, E)$ : if $\sigma \in G$ and $T \in \operatorname{End} \mathscr{Y}(X, E)$ then

$$
{ }^{\sigma} T(\phi)=\sigma\left[T\left(\sigma^{-1}(\phi)\right)\right], \quad \phi \in \mathscr{Y}(X, E) .
$$

The Galois group also permutes the unitary characters of $F^{+}$: if $\sigma \in \operatorname{Gal}(E / \mathbb{Q})$ and $\lambda$ is a unitary character of $F^{+}$then ${ }^{\sigma} \lambda$ is the character defined by

$$
{ }^{\sigma} \lambda(t)=\sigma(\lambda(t)), \quad t \in F^{+} .
$$

Let $\mathscr{P}$ be the topological closure of the prime ring of $F$. The image of $s \in \mathscr{P}^{*}$ in $\operatorname{Gal}(\mathbb{Q}(\lambda) / \mathbb{Q})$ under the canonical isomorphism of Lemma 2 will be denoted $\sigma_{s}$.

Lemma 9. Let $\sigma \in \operatorname{Gal}(E / \mathbb{Q})$. If $\left.\sigma\right|_{\mathbb{Q}(\lambda)}=\sigma_{s}$ then $^{\sigma} \lambda=\lambda[s]$.

Proof. (char $F=0$ ) Let $\mathfrak{i}$ be the conductor of $\lambda$. Given $t \in F$, fix $n \geq 1$ such that $t \in p^{-n} \mathfrak{i}$. Since $p^{n} t \in \mathfrak{i}$,

$$
1=\lambda\left(p^{n} t\right)=\lambda(t)^{p^{n}}
$$

thus $\lambda(t) \in v_{p^{n}}$. Fixing $r \in \mathbb{Z}$ such that $s \equiv r \bmod p^{n} \mathscr{P}$,

$$
\left({ }^{\sigma} \lambda\right)(t)=\sigma(\lambda(t))=\lambda(t)^{r}=\lambda(r t)=\lambda(s t),
$$

the last equality following from the fact $r t \equiv s t \bmod i$.

Given $\sigma \in \operatorname{Gal}(E / \mathbb{Q})$, let ${ }^{\sigma} W_{\lambda}$ be the projective representation defined by

$$
\left({ }^{\sigma} W_{\lambda}\right)(g)={ }^{\sigma}\left(W_{\lambda}(g)\right), \quad g \in \operatorname{Sp}(V) .
$$

Proposition 10. Let $\sigma \in \operatorname{Gal}(E / \mathbb{Q}(\sqrt{q}))$. If $\left.\sigma\right|_{\mathbb{Q}(\lambda)}=\sigma_{s}$ then ${ }^{\sigma} W_{\lambda}(g)=W_{\lambda[s]}(g)$.

The proof of Proposition 10 is based on the integral formula (3) and the following result: 
Lemma 11. Let $A$ be a totally disconnected topological group, $L \subseteq K$ an extension of fields, and $\mu$ a L-rational Haar measure on A. If $\sigma$ is an L-automorphism of $K$ then, for all $\phi \in \mathscr{Y}(A, K)$,

$$
\int_{A} \sigma(\phi) d \mu=\sigma\left(\int_{A} \phi d \mu\right)
$$
Proof. Using the notation introduced in the proof of Lemma 7, if $\phi=\sum_{i=1}^{k} c_{i} \chi_{A_{i}}$
then

$$
\sigma(\phi)=\sum_{i=1}^{k} \sigma\left(c_{i}\right) \chi_{A_{i}} .
$$

Therefore, since $\mu\left(A_{i}\right) \in L$ is fixed by $\sigma$,

$$
\begin{aligned}
\int \sigma(\phi) d \mu & =\sum_{i=1}^{k} \sigma\left(c_{i}\right) \mu\left(A_{i}\right)=\sum_{i=1}^{k} \sigma\left(c_{i}\right) \sigma\left(\mu\left(A_{i}\right)\right) \\
& =\sigma\left(\sum_{i=1}^{k} c_{i} \mu\left(A_{i}\right)\right)=\sigma\left(\int_{A} \phi d \mu\right) .
\end{aligned}
$$

Proof of Proposition 10. Let $g \in \mathrm{Sp}(V), \phi \in \mathscr{Y}(X, E)$, and $x \in X$. We assume $g$ has the matrix representation (2). Lemma 6 asserts that the measure $\mu_{\lambda, g}$ is $\mathbb{Q}(\sqrt{q})$-rational. Applying Lemma 11 to the case $A=Y_{g}, L=\mathbb{Q}(\sqrt{q}), K=E$, and $\mu=\mu_{\lambda, g}$, the definition of ${ }^{\sigma} W_{\lambda}$, the formula (3), and Lemma 9 yield

$$
\begin{aligned}
{\left[{ }^{\sigma} W_{\lambda}(g) \phi\right](x) } & \\
& =\sigma\left[W_{\lambda}(g)\left(\sigma^{-1} \phi\right)(x)\right] \\
& =\sigma\left[\int_{Y_{g}} \lambda\left(\frac{\langle x a, x b\rangle-2\langle x b, y c\rangle+\langle y c, y d\rangle}{2}\right)\left(\sigma^{-1} \phi\right)(x a+y c) d \mu_{\lambda, g} y\right] \\
& =\int_{Y_{g}} \sigma_{\lambda} \lambda\left(\frac{\langle x a, x b\rangle-2\langle x b, y c\rangle+\langle y c, y d\rangle}{2}\right) \phi(x a+y c) d \mu_{\lambda, g} y \\
& =\int_{Y_{g}} \lambda[s]\left(\frac{\langle x a, x b\rangle-2\langle x b, y c\rangle+\langle y c, y d\rangle}{2}\right) \phi(x a+y c) d \mu_{\lambda, g} y .
\end{aligned}
$$

Observing $s \in \mathscr{P}^{*} \subseteq \mathrm{O}^{*}$, Lemma 5 implies that $\mu_{\lambda[s], g}=\mu_{\lambda, g}$. The preceding calculation thus gives

$\left[{ }^{\sigma} W_{\lambda}(g) \phi\right](x)$

$$
\begin{aligned}
& =\int_{Y_{g}}\left[\lambda[s]\left(\frac{\langle x a, x b\rangle-2\langle x b, y c\rangle+\langle y c, y d\rangle}{2}\right) \phi(x a+y c)\right] d \mu_{\lambda[s], g} y \\
& =\left[W_{\lambda[s]}(g) \phi\right](x) .
\end{aligned}
$$




\section{Action of symplectic similitudes}

In the previous section, we described the action of Galois on the projective representations $W_{\lambda}$. Here, we discuss an action of the group of symplectic similitudes on the Weil representations.

Given $s \in F^{*}$, let $f_{s}$ be the element of $\mathrm{GL}(V)$ defined by

$$
(x+y) f_{s}=x+s y, \quad x \in X, y \in Y .
$$

Conjugation by $f_{s}$ leaves the symplectic group $\operatorname{Sp}(V)$ invariant. In fact, if $g \in$ $\operatorname{Sp}(V)$ is expressed in the matrix form (2) then

$$
g^{f_{s}}=\left(\begin{array}{cc}
a & s b \\
s^{-1} c & d
\end{array}\right) .
$$

In particular, we note that the spaces $Y_{g}$ and $Y_{g f_{s}}$ are equal, since $\operatorname{ker} c=\operatorname{ker} s^{-1} c$.

Lemma 12. If $s \in F^{*}$ then $\mu_{\lambda, g} f_{s}=|s|_{Y_{g}}^{-1 / 2} \mu_{\lambda, g}$.

Proof. Let $p_{i, s}, 0 \leq i \leq n$, be the elements of $\operatorname{Sp}(V)$ defined by

$$
\begin{aligned}
& x_{j} p_{i, s}= \begin{cases}s^{-1} x_{j} & \text { if } j \leq i, \\
x_{j} & \text { if } i<j,\end{cases} \\
& y_{j} p_{i, s}= \begin{cases}s y_{j} & \text { if } j \leq i, \\
y_{j} & \text { if } i<j .\end{cases}
\end{aligned}
$$

Note that $p_{i, s} \in P$ and

$$
\operatorname{det}\left(\left.p_{i, s}\right|_{Y}\right)=s^{i}
$$

Moreover, one readily verifies that

$$
\tau_{i}^{f_{s}}=\tau_{i} p_{i, s} .
$$

Let $g \in G$. If $g=p_{1} \tau_{i} p_{2}, p_{1}, p_{2} \in P$, then

$$
g^{f_{s}}=\left(p_{1} \tau_{i} p_{2}\right)^{f_{s}}=p_{1}^{f_{s}} \tau_{i}^{f_{s}} p_{2}^{f_{s}}=p_{1}^{f_{s}} \tau_{i}\left(p_{i, s} p_{2}^{f_{s}}\right) .
$$

Observing that both $p_{1}^{f_{s}}$ and $p_{i, s} p_{2}^{f_{s}}$ belong to $P$, (5) yields

$$
\mu_{\lambda, f^{f_{s}}}=\left|\operatorname{det}\left(\left.p_{1}^{f_{s}} p_{i, s} p_{2}^{f_{s}}\right|_{Y}\right)\right|^{-1 / 2} \overline{p_{1}^{f_{s}}} \cdot \mu_{\lambda, \tau_{i}} .
$$

Using (8), if $p \in P$ then $\left.p^{f_{s}}\right|_{Y}=\left.p\right|_{Y}$. As a consequence,

$$
\overline{p_{1}^{f_{s}}}=\bar{p}_{1}: Y_{g} \rightarrow Y_{\tau_{i}} \text {. }
$$

In light of these observations,

$\operatorname{det}\left(\left.p_{1}^{f_{s}} p_{i, s} p_{2}^{f_{s}}\right|_{Y}\right)=\operatorname{det}\left(\left.p_{1} p_{i, s} p_{2}\right|_{Y}\right)=\operatorname{det}\left(\left.p_{i, s}\right|_{Y}\right) \cdot \operatorname{det}\left(\left.p_{1} p_{2}\right|_{Y}\right)=s^{i} \operatorname{det}\left(\left.p_{1} p_{2}\right|_{Y}\right) ;$ 
hence

$$
\mu_{\lambda, g} f_{s}=\left|s^{i} \operatorname{det}\left(\left.p_{1} p_{2}\right|_{Y}\right)\right|^{-1 / 2} \bar{p}_{1} \cdot \mu_{\lambda, \tau_{i}}=|s|^{-i / 2} \mu_{\lambda, g}=|s|_{Y_{g}}^{-1 / 2} \mu_{\lambda, g},
$$

since $Y_{g}$ has dimension $i$ over $F$.

Let $W_{\lambda}^{f_{s}}$ be the projective representation of $\operatorname{Sp}(V)$ defined by

$$
W_{\lambda}^{f_{s}}(g)=W_{\lambda}\left(g^{f_{s}}\right) \text {. }
$$

For the proof of the next result, let $|\alpha|_{V}$ denote the module of an automorphism $\alpha$ of an $F$-vector space $V$ [Weil 1974, I.2]. We have

$$
|\alpha|_{V}=|\operatorname{det} \alpha| \text {. }
$$

In particular, the module of left multiplication by $s \in F^{*}$ on $V$ satisfies

$$
|s|_{V}=|s|^{\operatorname{dim} V} .
$$

Proposition 13. If $s \in F^{*}$ then $W_{\lambda}^{f_{s}}=W_{\lambda[s]}$.

Proof. Let $g \in \mathrm{Sp}(V)$. We assume that $g$ has the matrix representation (2), hence that of $g^{f_{s}}$ is given by (8). If $\phi \in \mathscr{S}(X)$ and $x \in X$ then the integral formula (3) and Lemma 12 yield

$$
\begin{aligned}
& {\left[W_{\lambda}\left(g^{f_{s}}\right) \phi\right](x)} \\
& =\int_{Y_{g} f_{s}} \lambda\left(\frac{\langle x a, s x b\rangle-2\left\langle s x b, s^{-1} y c\right\rangle+\left\langle s^{-1} y c, y d\right\rangle}{2}\right) \phi\left(x a+s^{-1} y c\right) d \mu_{\lambda, g} f_{s} y \\
& =|s|_{Y_{g}}^{-1 / 2} \int_{Y_{g}} \lambda\left(\frac{\langle x a, s x b\rangle-2\left\langle s x b, s^{-1} y c\right\rangle+\left\langle s^{-1} y c, y d\right\rangle}{2}\right) \phi\left(x a+s^{-1} y c\right) d \mu_{\lambda, g} y .
\end{aligned}
$$

Replacing $y$ by $s y$, the definition of $|s|_{Y_{g}}$ and Lemma 5 yield

$$
\begin{aligned}
& {\left[W_{\lambda}\left(g^{f_{s}}\right) \phi\right](x)} \\
& \quad=|s|_{Y_{g}}^{-1 / 2}|s|_{Y_{g}} \int_{Y_{g}} \lambda\left(\frac{\langle x a, s x b\rangle-2\langle s x b, y c\rangle+\langle y c, s y d\rangle}{2}\right) \phi(x a+y c) d \mu_{\lambda g} y \\
& \quad=|s|_{Y_{g}}^{1 / 2} \int_{Y_{g}} \lambda\left(s \cdot \frac{\langle x a, x b\rangle-2\langle x b, y c\rangle+\langle y c, y d\rangle}{2}\right) \phi(x a+y c) d \mu_{\lambda, g} y \\
& \quad=|s|_{Y_{g}}^{1 / 2} \int_{Y_{g}} \lambda[s]\left(\frac{\langle x a, x b\rangle-2\langle x b, y c\rangle+\langle y c, y d\rangle}{2}\right) \phi(x a+y c) d \mu_{\lambda, g} y \\
& \quad=\int_{Y_{g}} \lambda[s]\left(\frac{\langle x a, x b\rangle-2\langle x b, y c\rangle+\langle y c, y d\rangle}{2}\right) \phi(x a+y c) d \mu_{\lambda[s], g} y \\
& =\left[W_{\lambda[s]}(g) \phi\right](x) .
\end{aligned}
$$

This completes the proof of the proposition. 


\section{The fundamental identity}

Let

$$
\mathfrak{G}=\left\{\sigma \in \operatorname{Gal}(E / \mathbb{Q}(\sqrt{q})): \exists s \in \mathbb{O}^{*} \text { such that }\left.\sigma\right|_{\mathbb{Q}(\lambda)}=\sigma_{s^{2}}\right\} .
$$

Note that $\mathfrak{G}$ is a subgroup of $\operatorname{Gal}(E / \mathbb{Q}(\sqrt{q}))$. Given $s \in F^{*}$, let $g_{s} \in \operatorname{Sp}(V)$ be the map defined by

$$
(x+y) g_{s}=s^{-1} x+s y, \quad x \in X, y \in Y .
$$

We observe that $g_{s}$ lies in the parabolic subgroup $P$ that leaves $Y$ invariant and is related to the operator $f_{s^{2}}$ defined earlier by the identity

$$
f_{s^{2}}=s I \circ g_{s} .
$$

Proposition 14. Let $\sigma \in \mathfrak{G}$ and $g \in \operatorname{Sp}(V)$. If $\left.\sigma\right|_{\mathbb{Q}(\lambda)}=\sigma_{s^{2}}, s \in \mathcal{O}^{*}$, then

$$
{ }^{\sigma} W_{\lambda}(g)=W_{\lambda}\left(g_{s}\right)^{-1} W_{\lambda}(g) W_{\lambda}\left(g_{s}\right) .
$$

Proof. In light of Propositions 10 and 13,

$$
{ }^{\sigma} W_{\lambda}(g)=W_{\lambda\left[s^{2}\right]}(g)=W_{\lambda}^{f_{s^{2}}}(g)=W_{\lambda}\left(g^{f_{s^{2}}}\right)=W_{\lambda}\left(g^{g_{s}}\right) .
$$

Applying Theorem 4(i) with $p_{1}^{-1}=p_{2}=g_{s}$,

$$
W_{\lambda}\left(g^{g_{s}}\right)=W_{\lambda}\left(g_{s}^{-1}\right) W_{\lambda}(g) W_{\lambda}\left(g_{s}\right)=W_{\lambda}\left(g_{s}\right)^{-1} W_{\lambda}(g) W_{\lambda}\left(g_{s}\right) .
$$

This completes the proof of the proposition.

Corollary. If $t \in F^{*}$ and $\sigma \in \mathfrak{G}$ then ${ }^{\sigma} W_{\lambda}\left(g_{t}\right)=W_{\lambda}\left(g_{t}\right)$.

Proof. Fix $s \in \mathbb{O}^{*}$ such that $\left.\sigma\right|_{\mathbb{Q}(\lambda)}=\sigma_{s^{2}}$. Observing that $g_{s}$ and $g_{t}$ are commuting elements of $P$, the preceding proposition combines with Theorem 4(i) to yield

$$
{ }^{\sigma} W_{\lambda}\left(g_{t}\right)=W_{\lambda}\left(g_{s}\right)^{-1} W_{\lambda}\left(g_{t}\right) W_{\lambda}\left(g_{s}\right)=W_{\lambda}\left(g_{s}^{-1} g_{t} g_{s}\right)=W_{\lambda}\left(g_{t}\right),
$$

as required.

\section{The cocycle}

Our aim in this section is the construction of a 1-cocycle $\delta$ on

$$
\mathfrak{H}=\operatorname{Gal}\left(E / E_{0}\right)
$$

with values in $\operatorname{GL}(\mathscr{Y}(X, E))$ satisfying the identity (I):

$$
{ }^{\sigma} W_{\lambda}(g)=\delta(\sigma)^{-1} W_{\lambda}(g) \delta(\sigma), \quad g \in \operatorname{Sp}(V), \sigma \in \mathfrak{H} .
$$

When combined with restriction to $\mathbb{Q}(\lambda)$, the canonical isomorphism of Lemma 2 yields

$$
\left.\mathfrak{H} \simeq \operatorname{Gal}\left(\mathbb{Q}(\lambda) / E_{0} \cap \mathbb{Q}(\lambda)\right)\right) \simeq\left(F^{*}\right)^{2} \cap \mathscr{P}^{*}
$$


Let

$$
o= \begin{cases}2(p-1) & \text { if } q \text { is a square, } \\ p-1 & \text { if } q \text { is not a square, }\end{cases}
$$

and fix a primitive $o$-th root of unity $\epsilon \in F^{*}$. Furthermore, let

$$
U_{1}= \begin{cases}\{1\} & \text { if } \operatorname{char} F=p \\ \{r \in \mathscr{P}: r \equiv 1 \bmod p\} & \text { if } \operatorname{char} F=0\end{cases}
$$

Since $p$ is odd, the map $r \mapsto r^{2}$ is an automorphism of the pro- $p$ group $U_{1}$. This allows us to conclude that

$$
\left(F^{*}\right)^{2} \cap \mathscr{P}^{*}=\left\langle\epsilon^{2}\right\rangle \times U_{1} .
$$

The isomorphism (9) identifies $U_{1}$ with $\operatorname{Gal}\left(E / \mathbb{Q}\left(v_{p}, \sqrt{-1}\right)\right)$, where $v_{p}$ is the group of complex $p$-th roots of unity. This in turn leads to an identification of $\left\langle\epsilon^{2}\right\rangle$ with

$$
\mathfrak{H} / \operatorname{Gal}\left(E / \mathbb{Q}\left(v_{p}, \sqrt{-1}\right)\right) \simeq \operatorname{Gal}\left(\mathbb{Q}\left(v_{p}, \sqrt{-1}\right) / E_{0}\right) .
$$

In particular, the element $\eta$ of $\mathfrak{H}$ characterized by

$$
\left.\eta\right|_{\mathbb{Q}(\lambda)}=\sigma_{\epsilon^{2}}
$$

has order $o / 2$ and restricts to a generator of $\operatorname{Gal}\left(\mathbb{Q}\left(v_{p}, \sqrt{-1}\right) / E_{0}\right)$.

Given $\sigma \in \mathfrak{H}$, there is a unique integer $i, 1 \leq i \leq o / 2$, and a unique element $s \in U_{1}$, such that

$$
\left.\sigma\right|_{\mathbb{Q}(\lambda)}=\sigma_{\epsilon^{2 i} s^{2}}
$$

If $\tau$ is a second element of $\mathfrak{H}$, say

$$
\left.\tau\right|_{\mathbb{Q}(\lambda)}=\sigma_{\epsilon^{2 j} t^{2}}, \quad 1 \leq j \leq o / 2, \quad t \in U_{1},
$$

then

$$
\left.\sigma \tau\right|_{\mathbb{Q}(\lambda)}=\sigma_{\epsilon^{2 k}(s t)^{2}}
$$

where $s t \in U_{1}$ and

$$
k= \begin{cases}i+j & \text { if } i+j \leq o / 2 \\ i+j-o / 2 & \text { if } i+j>o / 2\end{cases}
$$

Our initial attempt at the construction of the cocycle is to define

$$
D(\sigma)=W_{\lambda}\left(g_{\epsilon^{i} s}\right),\left.\quad \sigma\right|_{\mathbb{Q}(\lambda)}=\sigma_{\epsilon^{2 i} s^{2}}, \quad 1 \leq i \leq o / 2, \quad s \in U_{1} .
$$

Proposition 14 ensures that

$$
{ }^{\sigma} W_{\lambda}(g)=D(\sigma)^{-1} W_{\lambda}(g) D(\sigma), \quad g \in \operatorname{Sp}(V), \sigma \in \mathfrak{H} .
$$


Assuming $\sigma$ and $\tau$ are as above, the definition of $D$ yields

$$
D(\sigma \tau)=W_{\lambda}\left(g_{\epsilon^{k} s t}\right)
$$

On the other hand, the Corollary to Proposition 14 gives

$$
{ }^{\sigma} D(\tau)={ }^{\sigma} W_{\lambda}\left(g_{\epsilon^{j} t}\right)=W_{\lambda}\left(g_{\epsilon^{j} t}\right),
$$

hence Theorem 4(i) yields

$$
D(\sigma)^{\sigma} D(\tau)=W_{\lambda}\left(g_{\epsilon^{i} s}\right) W_{\lambda}\left(g_{\epsilon^{j} t}\right)=W_{\lambda}\left(g_{\epsilon^{i+j} s t}\right) .
$$

If $i+j \leq o / 2$ then

$$
W_{\lambda}\left(g_{\epsilon^{i+j} s t}\right)=W_{\lambda}\left(g_{\epsilon^{k} s t}\right) .
$$

If $i+j>o / 2$ then, observing $\epsilon^{o / 2}=-1$, Theorem 4(i) yields

$$
W_{\lambda}\left(g_{\epsilon^{i+j} s t}\right)=W_{\lambda}\left(g_{-\epsilon^{k} s t}\right)=W_{\lambda}(\iota) W_{\lambda}\left(g_{\epsilon^{k} s t}\right),
$$

where $\iota=g_{-1}$ is the central involution of $\operatorname{Sp}(V)$ that maps $v \in V$ to $-v$. In summary,

$$
D(\sigma)^{\sigma} D(\tau)= \begin{cases}D(\sigma \tau) & \text { if } i+j \leq o / 2 \\ W_{\lambda}(\iota) D(\sigma \tau) & \text { if } i+j>o / 2\end{cases}
$$

In particular, $D$ is not a 1-cocycle; to get one we must account for the factor $W_{\lambda}(\iota)$. Since $\iota \in P$, Theorem 4(ii) implies that if $\phi$ belongs to $\mathscr{Y}(X, E)$ then

$$
\left[W_{\lambda}(\iota) \phi\right](x)=\phi(-x), \quad x \in X .
$$

In particular, $W_{\lambda}(\iota)$ is an involution, hence the operators

$$
\rho_{e}=\frac{1}{2}\left(I+W_{\lambda}(\iota)\right) \quad \text { and } \quad \rho_{o}=\frac{1}{2}\left(I-W_{\lambda}(\iota)\right)
$$

are orthogonal idempotents. Furthermore, recalling $\iota=g_{-1}$, the Corollary to Proposition 14 shows that both $\rho_{e}$ and $\rho_{o}$ are fixed by the action of Galois. Finally, since $I=\rho_{e}+\rho_{o}$, it is easily verified that the operators

$$
\rho_{e}+c \rho_{o}, \quad c \in E, c \neq 0,
$$

are invertible.

Lemma 15. The norm equation

$$
N(u)=-1, \quad N: \mathbb{Q}\left(v_{p}, \sqrt{-1}\right) \rightarrow E_{0}
$$

has a solution. 
Proof. The case $p \equiv 1 \bmod 4$ is covered by [Cliff et al. 2004, Lemma 24], an application of the Hasse Norm Theorem. Suppose $p \equiv 3 \bmod 4$. If $q$ is not a square then the extension $\mathbb{Q}\left(v_{p}, \sqrt{-1}\right) / E_{0}$ has odd degree $(p-1) / 2$, hence -1 is a solution of the norm equation. If $q$ is square then the extension has degree $p-1 \equiv 2 \bmod 4$. In this case, $\sqrt{-1} \in E_{0}$ is a solution.

Let $u$ be a solution of the norm equation of the preceding lemma. Given $\sigma \in \mathfrak{H}$, set

$$
A(\sigma)=\rho_{e}+\left(\prod_{l=0}^{i-1} \eta^{l}(u)\right) \rho_{0}, \quad \text { where }\left.\sigma\right|_{\mathbb{Q}(\lambda)}=\sigma_{\epsilon^{2 i} s^{2}}, \quad 1 \leq i \leq o / 2, \quad s \in U_{1}
$$

where $\eta$ satisfies (10). The remarks preceding Lemma 15 ensure that $A(\sigma) \in$ $\operatorname{GL}(\mathscr{Y}(X, E))$. With the notation introduced earlier, if $\sigma$ and $\tau$ belong to $\mathfrak{H}$ then

$$
A(\sigma \tau)=\rho_{e}+\left(\prod_{l=0}^{k-1} \eta^{l}(u)\right) \rho_{0} .
$$

On the other hand, observing

$$
\left.\sigma \eta^{-i}\right|_{\mathbb{Q}(\lambda)}=\sigma_{\epsilon^{2 i} s^{2}} \sigma_{\epsilon^{2}}^{-i}=\sigma_{\epsilon^{2 i} s^{2}} \sigma_{\epsilon^{-2 i}}=\sigma_{s^{2}},
$$

the fact (9) identifies $U_{1}$ with $\operatorname{Gal}\left(E / \mathbb{Q}\left(v_{p}, \sqrt{-1}\right)\right)$ allows us to deduce that the restrictions of $\sigma$ and $\eta^{i}$ to $\mathbb{Q}\left(v_{p}, \sqrt{-1}\right)$ coincide. Therefore,

$$
\begin{aligned}
{ }^{\sigma} A(\tau) & ={ }^{\sigma}\left[\rho_{e}+\left(\prod_{l=0}^{j-1} \eta^{l}(u)\right) \rho_{0}\right]=\rho_{e}+\left(\prod_{l=0}^{\sigma-1} \eta^{l}(u)\right) \rho_{0} \\
& =\rho_{e}+{ }^{\eta^{i}}\left(\prod_{l=0}^{j-1} \eta^{l}(u)\right) \rho_{0}=\rho_{e}+\left(\prod_{l=i}^{i+j-1} \eta^{l}(u)\right) \rho_{0}
\end{aligned}
$$

hence

$$
\begin{aligned}
A(\sigma)^{\sigma} A(\tau) & =\left[\rho_{e}+\left(\prod_{l=0}^{i-1} \eta^{l}(u)\right) \rho_{0}\right]\left[\rho_{e}+\left(\prod_{l=i}^{i+j-1} \eta^{l}(u)\right) \rho_{0}\right] \\
& =\left[\rho_{e}+\left(\prod_{l=0}^{i+j-1} \eta^{l}(u)\right) \rho_{0}\right] .
\end{aligned}
$$

If $i+j \leq o / 2$ then

$$
\prod_{l=0}^{i+j-1} \eta^{l}(u)=\prod_{l=0}^{k-1} \eta^{l}(u)
$$


hence

$$
A(\sigma)^{\sigma} A(\tau)=A(\sigma \tau) .
$$

If $i+j>o / 2$ then the choice of $\eta$ and $u$ yield

$$
\prod_{l=0}^{i+j-1} \eta^{l}(u)=\left(\prod_{l=0}^{(o-2) / 2} \eta^{l}(u)\right)\left(\prod_{l=o / 2}^{i+j-1} \eta^{l}(u)\right)=N(u) \prod_{l=0}^{k-1} \eta^{l}(u)=-\prod_{l=0}^{k-1} \eta^{l}(u) .
$$

Observing that $\rho_{e}=\rho_{e} W_{\lambda}(\iota)$ and $-\rho_{o}=\rho_{o} W_{\lambda}(\iota)$,

$$
A(\sigma)^{\sigma} A(\tau)=\rho_{e}-\left(\prod_{l=0}^{k-1} \eta^{l}(u)\right) \rho_{0}=\left[\rho_{e}+\left(\prod_{l=0}^{k-1} \eta^{l}(u)\right) \rho_{0}\right] W_{\lambda}(\iota)=A(\sigma \tau) W_{\lambda}(\iota) .
$$

In summary,

$$
A(\sigma)^{\sigma} A(\tau)= \begin{cases}A(\sigma \tau) & \text { if } i+j \leq o / 2 \\ A(\sigma \tau) W_{\lambda}(\iota) & \text { if } i+j>o / 2\end{cases}
$$

Consider the map $\delta: \mathfrak{H} \rightarrow \operatorname{GL}(\mathscr{Y}(X, E))$ given by

$$
\delta(\sigma)=A(\sigma) D(\sigma) .
$$

If $\sigma, \tau \in \mathfrak{H}$ are as above

$$
\delta(\sigma)^{\sigma} \delta(\tau)=(A(\sigma) D(\sigma))^{\sigma}(A(\tau) D(\tau))=A(\sigma) D(\sigma)^{\sigma} A(\tau)^{\sigma} D(\tau) .
$$

By Theorem 4(ii), ${ }^{\sigma} A(\tau) \in E\left[W_{\lambda}(i)\right]$ commutes with $D(\sigma)=W_{\lambda}\left(g_{\epsilon^{i} s}\right)$, hence

$$
A(\sigma) D(\sigma)^{\sigma} A(\tau)^{\sigma} D(\tau)=A(\sigma)^{\sigma} A(\tau) D(\sigma)^{\sigma} D(\tau) .
$$

If $i+j>o / 2$ then (12) and (13) yield

$$
A(\sigma)^{\sigma} A(\tau) D(\sigma)^{\sigma} D(\tau)=A(\sigma \tau) W_{\lambda}(\iota) W_{\lambda}(\iota) D(\sigma \tau)=A(\sigma \tau) D(\sigma \tau) .
$$

Since this is trivially true if $i+j \leq o / 2$, we conclude

$$
\delta(\sigma)^{\sigma} \delta(\tau)=A(\sigma \tau) D(\sigma \tau)=\delta(\sigma \tau) .
$$

This shows that $\delta$ is a 1-cocycle. Furthermore, if $g \in \mathrm{Sp}(V)$ then Theorem 4(i) shows that $A(\sigma) \in E\left[W_{\lambda}(\iota)\right]$ commutes with $W_{\lambda}(g)$, hence (11) yields

$$
\begin{aligned}
\delta(\sigma)^{-1} W_{\lambda}(g) \delta(\sigma) & =(A(\sigma) D(\sigma))^{-1} W_{\lambda}(g) A(\sigma) D(\sigma) \\
& =D(\sigma)^{-1} A(\sigma)^{-1} W_{\lambda}(g) A(\sigma) D(\sigma) \\
& =D(\sigma)^{-1} W_{\lambda}(g) D(\sigma) \\
& ={ }^{\sigma} W_{\lambda}(g),
\end{aligned}
$$

which verifies that (I) is satisfied. 


\section{The triviality of the cocycle}

Let $\delta: \mathfrak{H} \rightarrow \operatorname{GL}(\mathscr{S}(X, E))$ be the 1-cocycle satisfying (I) constructed above.

Lemma 16. If $\phi \in \mathscr{Y}(X, E)$ then there exists an open subgroup $\mathfrak{K}$ of $\mathfrak{H}$ such that

$$
\delta(\sigma) \phi=\phi, \quad \sigma \in \mathfrak{K} .
$$

Proof. If char $F=p$ then $\mathfrak{H}$ is a finite discrete group, so one may take $\mathfrak{K}$ to be the trivial subgroup.

Assume char $F=0$. If $\mathfrak{X}$ is a lattice in $X$ then the subgroups

$$
p^{k} \mathfrak{X}, \quad k \in \mathbb{Z},
$$

form a local base at the origin. Therefore, given $x \in X$, there exist $i_{x} \in \mathbb{Z}$ such that $\phi$ is constant on the $\operatorname{coset} x+p^{i_{x}} \mathfrak{X}$. As the family $\left\{x+p^{i_{x}} \mathfrak{X}: x \in X\right\}$ is an open cover of $X$, there exists $x_{1}, \ldots, x_{m}$ in $X$ such that

Set

$$
\operatorname{supp} \phi \subseteq \bigcup_{j=1}^{m} x_{j}+p^{i_{x_{j}}} \mathfrak{X} .
$$

$$
i=\max \left\{i_{x_{1}}, \ldots, i_{x_{m}}\right\}
$$

and consider $x+p^{i} \mathfrak{X} \cap \operatorname{supp} \phi, x \in X$. If it is empty then the restriction of $\phi$ to the coset $x+p^{i} \mathfrak{X}$ is identically 0 . If not, there exists $j$ such that $x+p^{i} \mathfrak{X} \cap x_{j}+p^{i_{x_{j}}} \mathfrak{X}$ is nonempty, hence

$$
x+p^{i} \mathfrak{X} \subseteq x_{j}+p^{i_{x_{j}}} \mathfrak{X}
$$

by choice of $i$. The choice of $i_{x_{j}}$ thus ensures that the restriction of $\phi$ to $x+p^{i} \mathfrak{X}$ is the constant function with value $\phi\left(x_{j}\right)$. We conclude that $\phi$ is constant on the $p^{i} \mathfrak{X}$-cosets of $X$.

Let $\sigma \in \mathfrak{H}$. If $\left.\sigma\right|_{\mathbb{Q}(\lambda)}=\sigma_{r^{2}}, r \in U_{1}$, then by construction $\delta(\sigma)=W_{\lambda}\left(g_{r}\right)$. Observing

$$
g_{r}=\left(\begin{array}{cc}
r^{-1} \cdot 1_{X} & 0 \\
0 & r \cdot 1_{Y}
\end{array}\right) \in P,
$$

if $x \in X$ then Theorem 4(i) yields

$$
(\delta(\sigma) \phi)(x)=\left(W_{\lambda}\left(g_{r}\right) \phi\right)(x)=|r|^{-\operatorname{dim} X / 2} \lambda\left(\frac{\left\langle r^{-1} x, r x\right\rangle}{2}\right) \phi\left(r^{-1} x\right)=\phi\left(r^{-1} x\right),
$$

since $r$ is a unit and $\langle$,$\rangle is F$-bilinear and alternating. Fix $j \in \mathbb{Z}$ such that $i>j$ and

$$
\operatorname{supp} \phi \subseteq p^{j} \mathfrak{X} .
$$

If $x \notin p^{j} \mathfrak{X}$ then neither is $r^{-1} x$, so the choice of $j$ ensures that

$$
(\delta(\sigma) \phi)(x)=\phi\left(r^{-1} x\right)=0=\phi(x) .
$$


On the other hand, suppose $x \in p^{j} \mathfrak{X}$. In this case, if $r \equiv 1 \bmod p^{i-j}$ then

$$
r^{-1} x+p^{i} \mathfrak{X}=x+p^{i-j} p^{j} \mathfrak{X}+p^{i} \mathfrak{X}=x+p^{i} \mathfrak{X},
$$

hence the choice of $i$ ensures that

$$
(\delta(\sigma) \phi)(x)=\phi\left(r^{-1} x\right)=\phi(x) .
$$

In light of the preceding discussion,

$$
\mathfrak{K}=\left\{\sigma \in \mathfrak{H}:\left.\sigma\right|_{\mathbb{Q}(\lambda)}=\sigma_{r^{2}}, r \equiv 1 \bmod p^{i-j}\right\}=\operatorname{Gal}\left(E / \mathbb{Q}\left(v_{p^{i-j}}, \sqrt{-1}\right)\right)
$$

has the required properties.

Let $K / \mathrm{k}$ be a Galois extension and $M$ a $K$-vector space equipped with an semilinear action of the Galois group $\operatorname{Gal}(K / k)$ : if $\sigma \in \operatorname{Gal}(K / k), m \in M$ and $e \in K$ then

$$
\sigma(e m)=\sigma(e) \sigma(m)
$$

For such an action, the fixed-point set

$$
M^{\mathrm{Gal}(K / k)}=\{m \in M: m=\sigma(m) \text { for all } \sigma \in \operatorname{Gal}(K / k)\}
$$

is a $k$-vector space. The canonical action of $\operatorname{Gal}(K / k)$ on $K$ yields a semilinear action on the tensor product $K \otimes_{k} M^{\mathrm{Gal}(K / k)}$ :

$$
\sigma(e \otimes m)=\sigma(e) \otimes m, \quad \sigma \in \operatorname{Gal}(K / k), e \in E, m \in M^{\mathrm{Gal}(K / k)} .
$$

The action of Galois on $M$ is said to be smooth if the stabilizer of each $m \in M$ is open in $\operatorname{Gal}(K / k)$.

Proposition 17. [Galois Descent] If $M$ is a $K$-vector space equipped with a semilinear, smooth action of $\mathrm{Gal}(K / k)$ then the canonical map

$$
\psi: K \otimes_{k} M_{k} \rightarrow M
$$

is a $K$-linear isomorphism of $\mathrm{Gal}(K / k)$-modules.

Proof. The case $K=k_{s}$, the separable closure of $k$, is proved in [Borel 1991, AG.14.2]. The general case is proved using the same argument, mutatis mutandis.

Proposition 18. There exists $\alpha \in \mathrm{GL}(\mathscr{S}(X, E))$ such that

$$
\delta(\sigma)=\alpha^{-1 \sigma} \alpha, \quad \sigma \in \mathfrak{H} .
$$

Proof. The canonical action (7) of $\mathfrak{H}$ on $\mathscr{S}(X, E)$ is clearly semilinear. It is furthermore smooth, since each element of $\mathscr{S}(X, E)$ takes only finitely many values in $E$. 
On the other hand, since $\delta$ is a 1-cocycle, then

$$
(\sigma, \phi) \mapsto \delta(\sigma) \sigma(\phi), \quad \sigma \in \mathfrak{H}, \phi \in \mathscr{Y}(X, E),
$$

is also an action of $\mathfrak{H}$ on $\mathscr{S}(X, E)$, referred to as the twisted action by $\delta$. It is semilinear, since $\delta$ takes values in $\operatorname{GL}(\mathscr{Y}(X, E))$. Since the original action is smooth, if $\phi \in \mathscr{S}(X, E)$ then there exists an open subgroup $\mathfrak{H}_{1}$ such that

$$
\sigma(\phi)=\phi, \quad \sigma \in \mathfrak{H}_{1} .
$$

Furthermore, Lemma 16 asserts that there is an open subgroup $\mathfrak{K}$ of $\mathfrak{H}$ such that

$$
\delta(\sigma) \phi=\phi, \quad \sigma \in \mathfrak{K} .
$$

Therefore, if $\sigma \in \mathfrak{H}_{1} \cap \mathfrak{K}$ then

$$
\delta(\sigma) \sigma(\phi)=\delta(\sigma) \phi=\phi .
$$

This shows that the stabilizer of $\phi$ under the twisted action contains the open subgroup $\mathfrak{H}_{1} \cap \mathfrak{K}$. Since it is the union of its $\mathfrak{H}_{1} \cap \mathfrak{K}$-cosets, it follows that the stabilizer of $\phi$ under the twisted action is open. We conclude that the twisted action is smooth.

Using $\mathscr{S}(X, E)$ and ${ }_{\delta} \mathscr{S}(X, E)$ to denote the $\mathfrak{H}$-modules defined by the natural and twisted actions, respectively, Galois Descent asserts the existence of $E$-linear, $\mathfrak{H}$-equivariant isomorphisms

$$
{ }_{\delta} \mathscr{Y}(X, E) \simeq E \otimes_{E_{0} \delta} \mathscr{S}(X, E)^{\mathfrak{H}} \quad \text { and } \quad E \otimes_{E_{0}} \mathscr{Y}(X, E)^{\mathfrak{H}} \simeq \mathscr{Y}(X, E) .
$$

In particular,

$$
\operatorname{dim}_{E_{0} \delta} \mathscr{S}(X, E)^{\mathfrak{H}}=\operatorname{dim}_{E} \mathscr{S}(X, E)=\operatorname{dim}_{E_{0}} \mathscr{S}(X, E)^{\mathfrak{H}},
$$

so ${ }_{\delta} \mathscr{Y}(X, E)^{\mathfrak{H}}$ and $\mathscr{Y}(X, E)^{\mathfrak{H}}$ are $E_{0}$-isomorphic. As any such isomorphism extends by scalars to a E-linear, $\mathfrak{H}$-equivariant isomorphism

$$
E \otimes_{E_{0} \delta} \mathscr{S}(X, E)^{\mathfrak{H}} \simeq E \otimes_{E_{0}} \mathscr{Y}(X, E)^{\mathfrak{H}},
$$

we conclude that

$$
{ }_{\delta} \mathscr{S}(X, E) \simeq \mathscr{Y}(X, E) .
$$

Let $\alpha \in \operatorname{GL}(\mathscr{Y}(X, E))$ be a $\mathfrak{H}$-equivariant isomorphism ${ }_{\delta} \mathscr{S}(X, E) \rightarrow \mathscr{S}(X, E)$. If $\sigma \in \mathfrak{H}$ and $\phi \in \mathfrak{H}$ then the definition of the twisted action ensures that

$$
\alpha \delta(\sigma) \sigma(\phi)=\sigma(\alpha \phi)
$$

hence

$$
\delta(\sigma) \phi=\alpha^{-1} \alpha \delta(\sigma) \sigma\left(\sigma^{-1}(\phi)\right)=\alpha^{-1} \sigma\left(\alpha\left(\sigma^{-1}(\phi)\right)\right)=\alpha^{-1 \sigma} \alpha(\phi) .
$$




\section{Proof of the main theorem}

Fix $\alpha \in \mathrm{GL}(\mathscr{Y}(X, E))$ satisfying the conclusion of Proposition 18. In light of (9) and (14), if $\sigma \in \mathfrak{H}$ and $g \in \operatorname{Sp}(V)$ then

${ }^{\sigma}\left(\alpha W_{\lambda}(g) \alpha^{-1}\right)={ }^{\sigma} \alpha^{\sigma} W_{\lambda}(g)\left({ }^{\sigma} \alpha\right)^{-1}={ }^{\sigma} \alpha \delta(\sigma)^{-1} W_{\lambda}(g) \delta(\sigma)\left({ }^{\sigma} \alpha\right)^{-1}=\alpha W_{\lambda}(g) \alpha^{-1}$.

The compatibility of the Galois actions (6) and (7) allows us to deduce that the operators

$$
\alpha W_{\lambda}(g) \alpha^{-1}, \quad g \in \mathrm{Sp}(V)
$$

leave

$$
\mathscr{S}(X, E)^{\mathfrak{H}}=\mathscr{Y}\left(X, E^{\mathfrak{H}}\right)=\mathscr{Y}\left(X, E_{0}\right)
$$

invariant, hence provide a projective Weil representation realized over $E_{0}$.

\section{Optimality of the field $E_{0}$}

It is natural to ask if the field $E_{0}$ is optimal in the sense that the Weil representation $W_{\lambda}$ may not be realized over a proper subfield. To investigate this, fix a lattice $\mathscr{L}$ of $V$ on which the symplectic form $\langle$,$\rangle is nondegenerate and consider the K$-types of the Weil representation $W_{\lambda}$ obtained by restricting to the compact subgroup $\operatorname{Sp}(\mathscr{L})$ [Prasad 1998].

Given a natural number $k$, let $\Gamma_{k}$ denote that normal subgroup of $\operatorname{Sp}(\mathscr{L})$ consisting of those elements $g$ for which

$$
v g \equiv v \bmod \mathfrak{m}^{k} \mathscr{L}, \quad v \in \mathscr{L},
$$

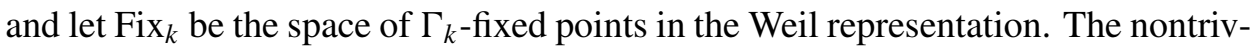
ial $K$-types of $W_{\lambda}$ associated with $\operatorname{Sp}(\mathscr{L})$ can be realized as the \pm 1 -eigenspaces of $\iota$, the central involution of $\operatorname{Sp}(V)$, acting on the quotients $\operatorname{Fix}_{2 i+2} /$ Fix $_{2 i}, i=0,1, \ldots$ Indeed, in light of Proposition 13 and the remarks preceding Proposition 14, it is sufficient to verify this when $\lambda$ has level 0 and -1 . The first case is an immediate consequence of the description of the $K$-types provided by [Prasad 1998, Theorem 2], while the second case follows from the analogous result for representations arising from characters of odd level. In particular, if $W_{\lambda}$ can be realized over a field $L$ then its $K$-types can also be realized over $L$.

The nontrivial $K$-types of $W_{\lambda}$ can be shown to coincide with the irreducible representations Top $^{ \pm}$studied in [Cliff et al. 2004]. If $q \equiv 1 \bmod 4$ then Top has Schur index 2, by Theorem 26 of that reference. Since Theorem 17 of the same work asserts that its character field is $\mathbb{Q}$ (respectively, $\mathbb{Q}(\sqrt{p})$ ) if $q$ is square (respectively, not square), Top ${ }^{-}$may not be realized over a proper subfield of $E_{0}$. The remarks made above allow us to conclude that $E_{0}$ is an optimal field for realizing $W_{\lambda}$. 
In the case $q \equiv 3 \bmod 4$, the representations $\mathrm{Top}^{ \pm}$all have Schur index 1 and character fields $\mathbb{Q}(\sqrt{-p})$ [Cliff et al. 2004, Theorems 17 and 26]. As a result, the restriction of $W_{\lambda}$ to the compact group $\operatorname{Sp}(\mathscr{L})$ can be realized over the subfield $\mathbb{Q}(\sqrt{-p})$ of $E_{0}$. The possibility of realizing the entire Weil representation over the field $\mathbb{Q}(\sqrt{-p})$ is left open.

\section{References}

[Borel 1991] A. Borel, Linear algebraic groups, 2nd ed., Graduate Texts in Mathematics 126, Springer, New York, 1991. MR 92d:20001 Zbl 0726.20030

[Cartier 1979] P. Cartier, "Representations of p-adic groups: a survey", pp. 111-155 in Automorphic forms, representations and L-functions (Corvallis, OR, 1977), edited by A. Borel and W. Casselman, Proc. Sympos. Pure. Math. 33, Amer. Math. Soc., Providence, R.I., 1979. MR 81e:22029 Zbl 0421.22010

[Cliff et al. 2004] G. Cliff, D. McNeilly, and F. Szechtman, "Character fields and Schur indices of irreducible Weil characters", J. Group Theory 7:1 (2004), 39-64. MR 2004m:20086 Zbl 1041.20030

[Kudla 1996] S. S. Kudla, "Notes on the local theta correspondence", lecture notes, 1996, available at http://www.math.toronto.edu/ skudla/castle.pdf.

[Mœglin et al. 1987] C. Mœglin, M.-F. Vignéras, and J.-L. Waldspurger, Correspondances de Howe sur un corps p-adique, Lecture Notes in Mathematics 1291, Springer, Berlin, 1987. MR 91f:11040 Zbl 0642.22002

[Prasad 1998] D. Prasad, "A brief survey on the theta correspondence", pp. 171-193 in Number theory (Tiruchirapalli, 1996), edited by V. K. Murty and M. Waldschmidt, Contemp. Math. 210, Amer. Math. Soc., Providence, RI, 1998. MR 99e:11063 Zbl 0922.11041

[Ranga Rao 1993] R. Ranga Rao, "On some explicit formulas in the theory of Weil representation", Pacific J. Math. 157:2 (1993), 335-371. MR 94a:22037 Zbl 0794.58017

[Weil 1974] A. Weil, Basic number theory, 2nd ed., Springer, New York, 1974. MR 55 \#302 Zbl 0326.12001

Received April 9, 2009. Revised August 18, 2011.

\section{GERALD CLIFF}

DePartment of Mathematical and Statistical Sciences

UNIVERSITY OF ALBERTA

EDMONTON, AB T6G 2G1

CANADA

gcliff@math.ualberta.ca

DaVid MCNEILly

Department of Mathematical and Statistical Sciences

UNIVERSITY OF ALBERTA

EDMONTON, AB T6G 2G1

CANADA

dam@math.ualberta.ca 


\title{
PACIFIC JOURNAL OF MATHEMATICS
}

\author{
http://pacificmath.org \\ Founded in 1951 by \\ E. F. Beckenbach (1906-1982) and F. Wolf (1904-1989)
}

\section{EDITORS}

V. S. Varadarajan (Managing Editor)

Department of Mathematics

University of California

Los Angeles, CA 90095-1555

pacific@math.ucla.edu

Vyjayanthi Chari

Department of Mathematics

University of California

Riverside, CA 92521-0135

chari@math.ucr.edu

\section{Robert Finn}

Department of Mathematics Stanford University

Stanford, CA 94305-2125

finn@math.stanford.edu

Kefeng Liu

Department of Mathematics

University of California

Los Angeles, CA 90095-1555

liu@math.ucla.edu
Darren Long

Department of Mathematics

University of California

Santa Barbara, CA 93106-3080

long@math.ucsb.edu

Jiang-Hua Lu

Department of Mathematics

The University of Hong Kong

Pokfulam Rd., Hong Kong jhlu@maths.hku.hk

Alexander Merkurjev

Department of Mathematics

University of California

Los Angeles, CA 90095-1555

merkurev@math.ucla.edu
Sorin Popa

Department of Mathematics University of California

Los Angeles, CA 90095-1555 popa@math.ucla.edu

Jie Qing

Department of Mathematics

University of California

Santa Cruz, CA 95064

qing@cats.ucsc.edu

Jonathan Rogawski

Department of Mathematics

University of California

Los Angeles, CA 90095-1555

jonr@math.ucla.edu

\section{PRODUCTION}

pacific@math.berkeley.edu

\section{SUPPORTING INSTITUTIONS}

ACADEMIA SINICA, TAIPEI

CALIFORNIA INST. OF TECHNOLOGY INST. DE MATEMÁTICA PURA E APLICADA KEIO UNIVERSITY

MATH. SCIENCES RESEARCH INSTITUTE NEW MEXICO STATE UNIV.

OREGON STATE UNIV.

\author{
STANFORD UNIVERSITY \\ UNIV. OF BRITISH COLUMBIA \\ UNIV. OF CALIFORNIA, BERKELEY \\ UNIV. OF CALIFORNIA, DAVIS \\ UNIV. OF CALIFORNIA, LOS ANGELES \\ UNIV. OF CALIFORNIA, RIVERSIDE \\ UNIV. OF CALIFORNIA, SAN DIEGO \\ UNIV. OF CALIF., SANTA BARBARA
}

\author{
UNIV. OF CALIF., SANTA CRUZ \\ UNIV. OF MONTANA \\ UNIV. OF OREGON \\ UNIV. OF SOUTHERN CALIFORNIA \\ UNIV. OF UTAH \\ UNIV. OF WASHINGTON \\ WASHINGTON STATE UNIVERSITY
}

These supporting institutions contribute to the cost of publication of this Journal, but they are not owners or publishers and have no responsibility for its contents or policies.

See inside back cover or pacificmath.org for submission instructions.

The subscription price for 2012 is US \$420/year for the electronic version, and \$485/year for print and electronic.

Subscriptions, requests for back issues from the last three years and changes of subscribers address should be sent to Pacific Journal of Mathematics, P.O. Box 4163, Berkeley, CA 94704-0163, U.S.A. Prior back issues are obtainable from Periodicals Service Company, 11 Main Street, Germantown, NY 12526-5635. The Pacific Journal of Mathematics is indexed by Mathematical Reviews, Zentralblatt MATH, PASCAL CNRS Index, Referativnyi Zhurnal, Current Mathematical Publications and the Science Citation Index.

The Pacific Journal of Mathematics (ISSN 0030-8730) at the University of California, c/o Department of Mathematics, 969 Evans Hall, Berkeley, CA 94720-3840, is published monthly except July and August. Periodical rate postage paid at Berkeley, CA 94704, and additional mailing offices. POSTMASTER: send address changes to Pacific Journal of Mathematics, P.O. Box 4163, Berkeley, CA 94704-0163.

PJM peer review and production are managed by EditFLOW ${ }^{\mathrm{TM}}$ from Mathematical Sciences Publishers.

PUBLISHED BY PACIFIC JOURNAL OF MATHEMATICS

at the University of California, Berkeley 94720-3840

A NON-PROFIT CORPORATION

Typeset in LATEX

Copyright $(02012$ by Pacific Journal of Mathematics 


\section{PACIFIC JOURNAL OF MATHEMATICS}

Volume $255 \quad$ No. $1 \quad$ January 2012

Averaging sequences

FERnANDo Alcalde Cuesta and Ana Rechtman

Affine group schemes over symmetric monoidal categories

ABHISHEK BANERJEE

Eigenvalue estimates on domains in complete noncompact Riemannian

manifolds

Daguang Chen, TAO Zheng and Min LU

Realizing the local Weil representation over a number field

GERALD CLIFF and DAVID MCNEILLY

Lagrangian submanifolds in complex projective space with parallel second fundamental form

Franki DiLlen, Haizhong Li, LuC VRANCKEN and XiANFENG

WANG

Ultra-discretization of the $D_{4}^{(3)}$-geometric crystal to the $G_{2}^{(1)}$-perfect crystals

Mana Igarashi, KaIlash C. MisRa and ToshiKi NAKASHIMA Connectivity properties for actions on locally finite trees

KEITH JONES

Remarks on the curvature behavior at the first singular time of the Ricci flow

NAM Q. LE and NATASA SESUm

Stability of capillary surfaces with planar boundary in the absence of gravity

PetKo I. MARINOV

Small hyperbolic polyhedra

SHAWN RAFALSKI

Hurwitz spaces of coverings with two special fibers and monodromy group 241 a Weyl group of type $B_{d}$

FRANCESCA VETRO 